Full Proceeding Paper

\title{
WAVELET TRANSFORMATION FOR ENHANCING MAMMOGRAPHIC IMAGES
}

\author{
ANKUSH RAI, JAGADEESH KANNAN R \\ School of Computing Science and Engineering, VIT University, Chennai, Tamil Nadu, India. Email: ankushressci@gmail.com
}

Received: 28 December 2016, Revised and Accepted: 10 May 2017

\begin{abstract}
Objective: Mammographic images are often prone to noises and consequently make the task of radiologist to come up with the precise diagnosis. Although there are several denoising techniques for the same is available, while denoising they often suffer from the problem of eliminating the micron level details in the noise influenced images. It's a trade-off which prohibits efficient micro-classification of mammary tissues.
\end{abstract}

Methods: In this study, we present a solution for the same by utilizing multi-level wavelet transformation to enable preservation of micron level details in the images.

Results and Conclusion: The quality denoising without elimination of the features of the mammographic imagery data by MWTA will allow the medical practitioner to easily identify and consequently diagnose properly to cancer influenced patients.

Keywords: Digital mammography, Wavelet transformation, Medical Image denoising.

(C) 2017 The Authors. Published by Innovare Academic Sciences Pvt Ltd. This is an open access article under the CC BY license (http://creativecommons. org/licenses/by/4. 0/) DOI: http://dx.doi.org/10.22159/ajpcr.2017.v10s1.19739

\section{INTRODUCTION}

Breast cancer is the common cancer among the women of 3555 age groups and is the leading cause of cancer deaths followed by lung cancers. Over more than two million women alone in the United States are diagnosed with the breast cancer treatment. Although the prevention against such deadly disease is impossible as the cause of this disease is still unknown, at the moment the early detection and prognosis/diagnosis is of vital steps in countering this disease. The imaging techniques such as X-ray mammography are primarily used in the detection of cancer. Here, in this scenario, the microclassification of clusters is the important sign of breast cancers [1]. In mammograms, this microclassifications appears as nodular points with high contrast or of high intensity localized diffusively along the breast.

However, there is a significant challenge in detection of early signs of breast cancers that appear on X-ray mammograms due to the major influence of various sorts of noises dictating the appearance of the final mammogram. These noises can be of the source of origin from malfunctioning equipment or from the faulty practices in recording the imagery data. This lead to the problem of identifying and detecting the breast area inflamed with cancer virus from the naked eyes. Thereby, making it suspicious to heavily rely on the noise corrupted imagery data surrounding the breast tissues; making essential regions invisible or mixed with the noises.

The past decades have witnessed various denoising techniques for mammographic image denoising; though they are often prone to manual intervention, poor performance, fail to preserve the features of the image after denoising. These methods are usually composed of a wide range of combination of fuzzy logic, wavelet transformation or that of the neural network [2-6]. Since the mammograms show larger areas of varying contrast and brightness, thus the information is highly susceptible to being correlated [7-10]. Other researchers used wavelet transformation to an extent where it tends to give more consolidated results than the other methods [11,12]; therefore, the following study presents an effectively modeled algorithm for multi-wavelet transformation to denoise the noisy mammographic images to allow easy microclassification to help doctors or radiologist detect breast cancer easily.

\section{METHODS}

\section{Experimental setup}

The proposed model is implemented using MATLAB R2012a under Windows platform. The experiments are conducted over the machine with hardware configurations of Intel's third generation 8-core microprocessor with Nvidea 630 graphic card, 2 GB RAM giving a fine clocking speed of $2.7 \mathrm{GHz}$. The consolidated database used in the study is digital database for screening mammography (DDSM) by the University of South Florida and is available online at [13]. The images used in the study consist of three types and are classified into three types based on the amount of cancer influenced the mammary tissue. The mammographic consists of 16 bits of information and with the resolution ranging from 42 to $43.5 \mu$. The images are extracted by the scanners namely DBA, HOWTEK, and LUMSIYS. These canners are deviated based on the optical density required to extract the information from the mammogram images. The properties of the used images are represented in Table 1.

The multi-wavelet transformation model

We employ the multi-wavelet transformation to breakdown the given noisy image into a pyramid of features which is linked to one another in logical manner. This will allow us to perform tree based searching and allocation for a given color scheme which will be independent from feature decomposition for both high and low-resolution image [14-16]. Therefore, the image can be broken into wavelets by using the following functions as given below:

$$
\begin{aligned}
f(x, y)= & \frac{1}{\sqrt{\mathrm{MN}}} \sum_{\mathrm{m}} \sum_{\mathrm{n}} \mathrm{w}_{\phi}\left(\mathrm{j}_{0}, \mathrm{~m}, \mathrm{n}\right) \phi_{\mathrm{j}_{\mathrm{o}}, \mathrm{m}, \mathrm{n}}(\mathrm{x}, \mathrm{y})+ \\
& \frac{1}{\sqrt{\mathrm{MN}}} \sum_{\mathrm{m}} \sum_{\mathrm{n}} \mathrm{w}_{\psi}^{\mathrm{i}}(\mathrm{j}, \mathrm{m}, \mathrm{n}) \psi_{\mathrm{j}, \mathrm{m}, \mathrm{n}}^{\mathrm{i}}(\mathrm{x}, \mathrm{y})
\end{aligned}
$$

Table 1: Enlisted database used in the experiment

\begin{tabular}{llll}
\hline Cancer influence & Size and bits & Resolutions $(\mu)$ & Scanners \\
\hline Normal & 6.6 GB 16 bits & 42 & DBA \\
Cancer & 6.6 GB 16 bits & 43.5 & HOWTEK \\
Benign & 6.1 GB 16 bits & 43.5 & LUMSIYS \\
\hline
\end{tabular}


Where, the indices $\mathrm{j}_{0}, \mathrm{j}, \mathrm{m}, \mathrm{n}$ are the non-negative integers, $\mathrm{x}$ and $\mathrm{y}$ are the pixels position at point $\mathrm{P}, \mathrm{M}$ and $\mathrm{N}$ are the real valued tensor coefficients, $\phi$ is the scaling function, and $\psi$ is the wavelet function in corresponding scaling and wavelet function is given by $W_{\phi^{\prime}}, W_{\psi}^{\mathrm{i}}$. The scaling coefficients from the given noisy image are at different resolution in a mammogram while the wavelet coefficients from the feature vector in the noise retrieval step; that's the reason why different types of scanners are used in recording the mammogram which in turn is dependent on the optical density.

During the sampling phase, the wavelet coefficients can be transformed into feature sets with the generalized association rule by formulating a Gaussian kernel based on the similarity of the coefficients and its characteristics from the given noisy image. The Gaussian kernel so formed s given as:

$k\left(x, x^{\prime}\right)=\frac{1}{N_{i}} \sum_{x, y \subset R_{t}}\left|I_{t}(x-1, y-1)-I_{t}(x+1, y+1)\right| e^{\left(-\frac{\left\|x-x^{\prime}\right\|^{2}}{2 \sigma}\right)}$

Where, $N_{i}$ is the total number of neighboring pixels in the spatial region of the pixel position $x, y$. Here, $R_{t}$ is the regularized threshold value, $I_{t}$ is the intensity of the pixel value for the diagonal of the pixel region, $\sigma$ is characterized by gradient descent of the standard deviation for a particular band at different scales of the mammogram, and x' is the next pixel position. Here, the emphasis is toward evaluating the kernel and updating it by pairing the formulation in association with one another. The flow chart of the work flow process involving the denoising process is given in Fig. 1. The threshold value of the wavelet to give a denoised image is determined as:

$$
\mathrm{R}_{\mathrm{t}}\left(\mathrm{x}, \mathrm{x}^{\prime}\right)=\left\{\begin{array}{c}
\mathrm{W}_{\phi} * \mathrm{~W}_{\psi}^{\mathrm{i}}, \text { if } \sigma^{*} \phi>\psi \\
0, \sigma^{*} \phi \leq \psi
\end{array}\right.
$$

Algorithm: Multi-wavelet transformation algorithm (MWTA) $\mathrm{x}^{\prime}$

Input: Noisy mammographic image I (Fig. 2)

Output: Denoised mammographic image I'

Step 1: Read the input noisy mammographic image I and use multiwavelet transformation (Fig. 3) to break down the given noisy image into a pyramid of features which is linked to one another in logical manner as:

$$
\begin{aligned}
f(x, y)= & \frac{1}{\sqrt{\mathrm{MN}}} \sum_{\mathrm{m}} \sum_{\mathrm{n}} \mathrm{w}_{\phi}\left(\mathrm{j}_{0}, \mathrm{~m}, \mathrm{n}\right) \phi_{\mathrm{j}_{0}, \mathrm{~m}, \mathrm{n}}(\mathrm{x}, \mathrm{y})+ \\
& \frac{1}{\sqrt{\mathrm{MN}}} \sum_{\mathrm{m}} \sum_{\mathrm{n}} \mathrm{w}_{\psi}^{\mathrm{i}}(\mathrm{j}, \mathrm{m}, \mathrm{n}) \psi_{\mathrm{j}, \mathrm{m}, \mathrm{n}}^{\mathrm{i}}(\mathrm{x}, \mathrm{y})
\end{aligned}
$$

Step 2: For each $(\mathrm{x}, \mathrm{y}) / /$ whole pixels of the given image.

Evaluate Gaussian kernel (Fig. 4) in combination with the neighboring diagonal pixels by:

$k\left(x, x^{\prime}\right)=\frac{1}{N_{i}} \sum_{x, y \subset R_{t}}\left|I_{t}(x-1, y-1)-I_{t}(x+1, y+1)\right| e^{\left(-\frac{\left\|x-x^{\prime}\right\|^{2}}{2 \sigma}\right)}$

Step 3: Determine threshold value

$\mathrm{R}_{\mathrm{t}}\left(\mathrm{x}, \mathrm{x}^{\prime}\right)=\left\{\begin{array}{c}\mathrm{W}_{\phi}{ }^{*} \mathrm{~W}_{\psi}^{\mathrm{i}}, \text { if } \sigma^{*} \phi>\psi \\ 0, \sigma^{*} \phi \leq \psi\end{array}\right.$

Step 4: end for

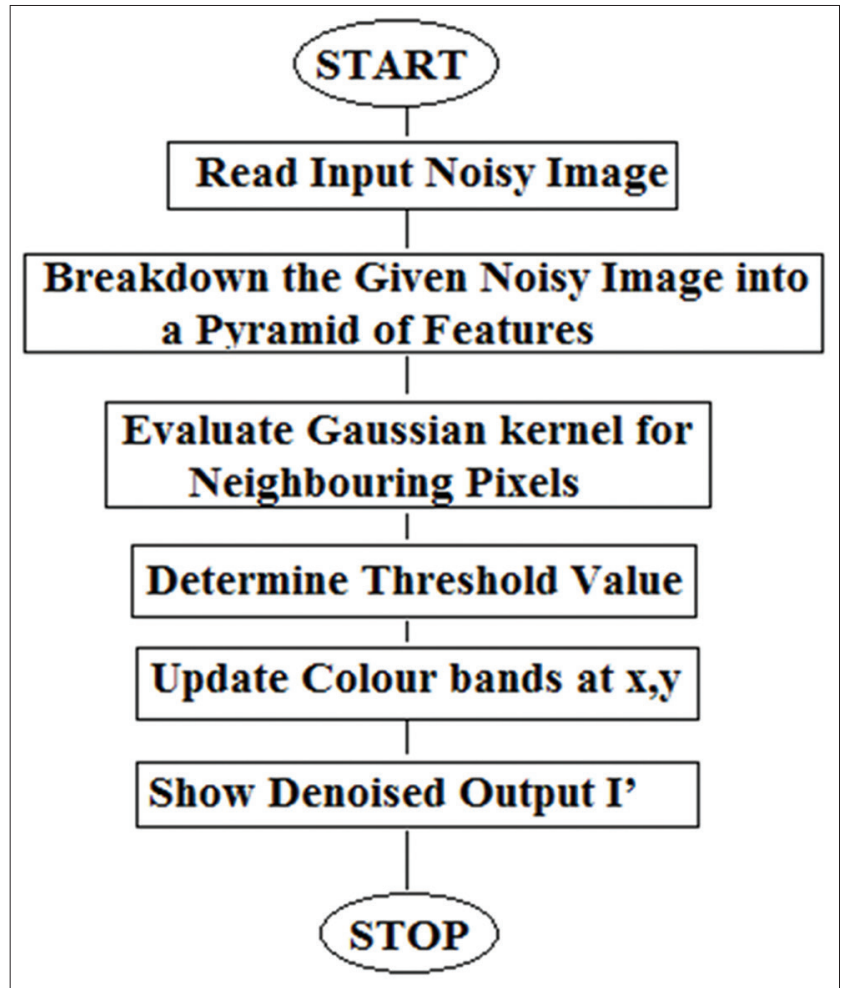

Fig. 1: Flow chart of the multi-wavelet transformation algorithm algorithm

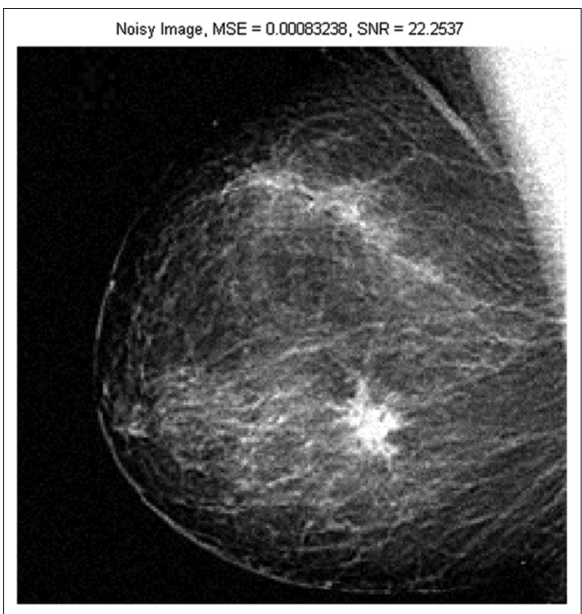

Fig. 2: Read input image I

Step 5: Update color bands at x,y and show output I' (Fig. 5).

Step 6: End process.

\section{CONCLUSION}

We have presented the quantifying success of the proposed algorithm against the three mostly used techniques for denoising the digital mammographic images. Table 2 represents the performance range of mammographic images with different amount of noise influence represented in the form of noise percentage for a DDSM database. Fig. 6 shows some of the samples of denoising results where mean signal error and signal to noise ratio are the two standard parameters used to compare the performance of denoising. The assessment of comparative performance results for the denoising methods with that of the MWTA algorithm suggests the affectivity of performance for the proposed method against the previous methods. The quality 


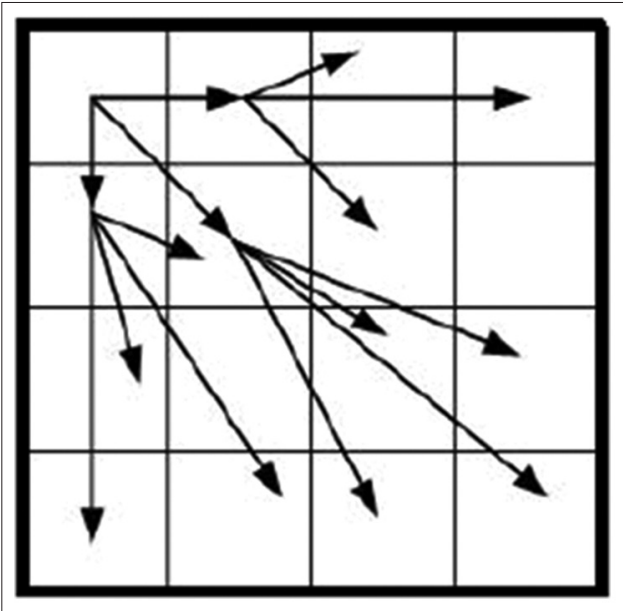

Fig. 3: Illustration of the decomposition of wavelets in form of logical manner by using the above equation

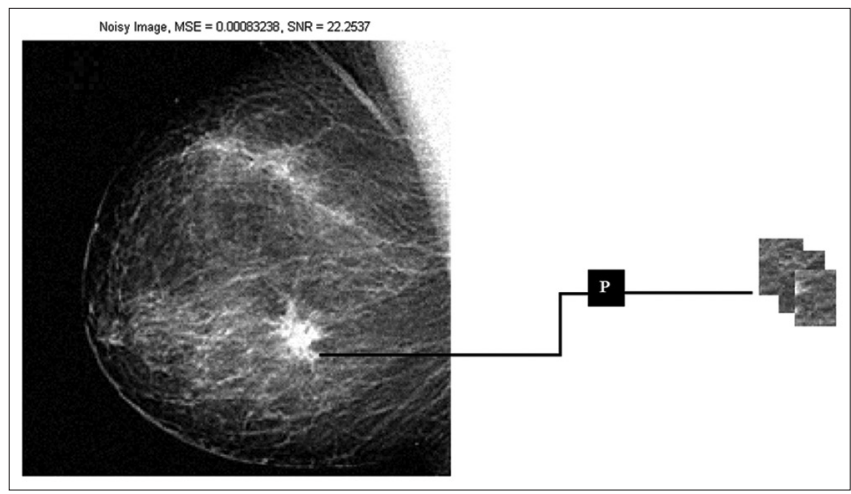

Fig. 4: Illustration of the formation of Gaussian kernels of wavelets localized in a neighboring region of the pixel position $\mathrm{x}, \mathrm{y}$

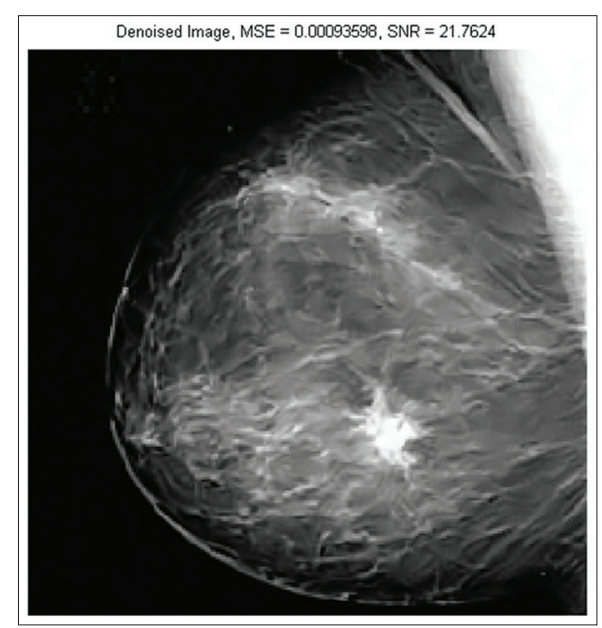

Fig. 5: Output denoised image I

Table 2: The tabular comparison results of the digital mammograms for different images from DDSM database

\begin{tabular}{lllll}
\hline Noise percentage (\%) & Wiener & Wavelet & ICA & MWTA \\
\hline 10.07 & 3.53 & 3.24 & 3.71 & 5.68 \\
25.05 & 15.97 & 13.62 & 14.05 & 17.89 \\
34.94 & 22.43 & 23.38 & 23.71 & 26.34 \\
45.28 & 33.33 & 33.79 & 34.28 & 37.12 \\
\hline
\end{tabular}

MWTA: Multi-wavelet transformation algorithm, ICA: Independent component analysis, DDSM: Digital database for screening mammography
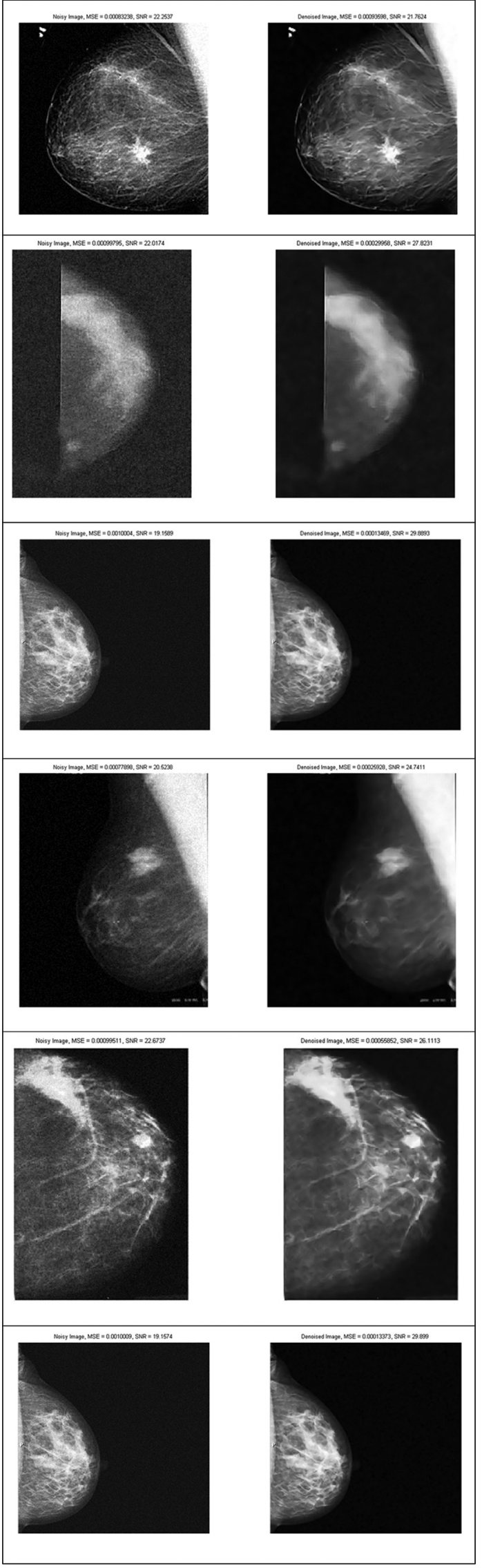

Fig. 6: Sample results of the denoised mammographic images from the digital database for screening mammography database 
denoising without elimination of the features of the mammographic imagery data by MWTA will allow the medical practitioner to easily identify and consequently diagnose properly to cancer influenced patients.

\section{REFERENCES}

1. Heinlein P, Drexl J, Schneider W. Integrated wavelet for enhancement of microcalcification in digital mammography. IEEE Trans Med Imaging 2000;22(3):402-13.

2. Pandey N, Salcic Z, Sivaswamy J. Fuzzy logic based microcalcification detection. Proceedings of the IEEE for Signal Processing Workshop; 2000. p. 662-71.

3. Strickland RN, Hahn HL. Wavelet transforms for detecting microcalcifications in mammograms. IEEE Trans Med Imaging 1996;15(2):218-29.

4. Mini MG, Thomas T. A neural network method for mammogram analysis based on statistical features. Proc TENCON 2003;4:1489-92.

5. Yu S, Guan L. A CAD system for the automatic detection of clustered microcalcifications in digitized mammogram films. IEEE Trans Med Imaging 2000;19(2):115-26.

6. Choubey A, Sinha GR, Choubey S. A hybrid filtering technique in medical image denoising: Blending of neural network and fuzzy inference. In: Electronics Computer Technology (ICECT), $20113^{\text {rd }}$ International Conference. Vol.1. p. 170,177. 8-10 April; 2011. DOI: 10.1109/ICECTECH.2011.5941584.

7. Ferreira CB, Borges DL. Analysis of mammogram classification using a wavelet transform decomposition. Pattern Recognit Lett 2003;24:973-82

8. Sentelle S, Sentelle C, Sutton MA. Multiresolution-based segmentation of calcifications for the early detection of breast cancer. Real-Time Imaging 2002;8:237-52

9. Nakayama R, Uchiyama Y, Yamamoto K, Watanabe R, Namba K. Computer-aided diagnosis scheme using a filter bank for detection of microcalcification clusters in mammograms. IEEE Trans Biomed Eng 2006;53(2):273-83.

10. Zhang PB. Noise reduction for magnetic resonance images via adaptive multiscale products thresholding. IEEE Trans Med Imaging 2003;22(9):1089-99.

11. Sameti M, Ward RK, Morgan-Parkes J, Palcic B. Image feature extraction in the last screening mammograms prior to detection of breast cancer. IEEE J Sel Top Signal Process 2009;3(1):46-52.

12. Metz CE. Some practical issues of experimental design and data analysis in radiological ROC studies. Invest Radiol 1989;24(3):234-45.

13. University of South Florida Digital Mammography, DDSM: Digital Database for Screening Mammography. Available from: http://www. marathon.csee.usf.edu/Mammography/Database.html.

14. Rai A. Attribute based level adaptive thresholding algorithm for object extraction. J Adv Robotics 2015;1(2):64-8.

15. Rai A. A novel decomposable pixel component analysis algorithm for automating multispectral satellite image denoising. Res Rev J Embed Syst Appl 2015;2(3):18-25

16. Rai A. High performance computing: A reality at Central-India. Int J Innov Res Dev 2013;2(3):734-43. 